\title{
Effect of highly active anti-retroviral treatment on TB incidence among HIV infected children and their clinical profile, retrospective Cohort study, South West Ethiopia
}

Firew Tiruneh Tiyare ( $\nabla$ mtu2012x@gmail.com )

Mizan-Tepi University https://orcid.org/0000-0001-5170-7610

Yared Deyas Deyas

Mizan-Tepi University

Research article

Keywords: TB incidence, HAART, Clinical Profile

Posted Date: March 5th, 2020

DOI: https://doi.org/10.21203/rs.3.rs-16218/v1

License: (c) (i) This work is licensed under a Creative Commons Attribution 4.0 International License.

Read Full License 


\section{Abstract}

Background: Children younger than 15 years, carries almost $80 \%$ of the global burden of HIV/ AIDS. Nearly, $50 \%$ of cases of tuberculosis are attributed to HIV infection. HIV worsen the progression of latent TB to active TB disease. Despite antiretroviral treatment has shown marked reduction in Tuberculosis incidence, TB continues to occur in Sub Saharan country including Ethiopia. The effect of highly active antiretroviral treatment is quite successful in developed country while in developing country TB/HIV coinfection remains perplexing among children on the treatment. The aim of the study was to investigate the impact of ART on the incidence of tuberculosis among Children infected with HIV in southwest Ethiopia. Methods: A retrospective cohort study was conducted on randomly selected 800 samples from ART clinic; between 2009 to 2014. We used chi-square test, and Mann-WhitneyU test to compare HAART naïve and HAART cohort. We used marginal structural models to estimate the effect of HAART on survival while accounting for time-dependent confounders affected by exposure. Result: A total of 844 children were followed for 2942.99 child-years. The children were observed for a median of 51 months with IQR 31 and for a total of 2942.99 child-years. From 506 Ols that occurred, the most common reported Ols were Pneumonia (22\%) and TB (23.6\%). The overall TB incidence rate was 7.917 per 100 child years $(95 \% \mathrm{Cl}$, 6.933-9.002). Whereas among HAART (7.667 per 100 -years (95\% Cl, 6.318-9.217) and 8.1686 per 100 person-years (95\% $\mathrm{Cl} 6.772-9.767)$ for HAART naïve. The mortality hazard ratio comparing HAART with no HAART from a marginal structural model was $0.642(95 \% \mathrm{Cl} 0.442-0.931, \mathrm{p}<0.02)$ Conclusions: HAART reduced the hazard of TB in HIV-infected children by $36 \%$. This is by far less than expected. Key WordsTB incidence, HAART, Clinical Profile ted children by $36 \%$. This is by far less than expected. Key Words- TB incidence, HAART, Clinical Profile

\section{Introduction}

Children younger than 15 years, carries almost $80 \%$ of the global burden of HIV/ AIDS. Infection with HIV is an important risk factor for Tuberculosis(1).Worldwide TB is the most common opportunistic illness and the leading cause of death among HIV infected children(2)(3). A total of 1.5 million people died from TB in 2018 (including 251000 people with HIV). In the same year, 1.1 million children fell ill with TB globally, and there were $20 \%$ child deaths due to TB (including among children with HIV)(4). According to WHO, there were an estimated 0.9 million new cases of TB amongst people who were HIV-positive, $72 \%$ of whom were living in Africa(3). Around $15-20 \%$ of TB cases were estimated among children in settings with a high burden of $\mathrm{TB}(5)(6)$.

HIV and TB form a lethal combination, each speeding the other's progress(7)(8). People with Latent TB have lifetime risk of $10-15 \%$ to develop active TB disease. The risk of developing TB in HIV-infected children is 20 times higher than HIV-uninfected children(9). TB risk is higher among HIV infected children. Without treatment, $15-50 \%$ of HIV-positive infants and children will develop active TB within two years after becoming infected with $\mathrm{TB}(10)$. 
To prevent TB, combined antiretroviral therapy (CART) against human immunodeficiency virus (HIV) is one of the strategies recommended by the World Health Organization (WHO) to prevent tuberculosis(11) (12). HAART has shown marked reduction in the incidence of Tuberculosis(1)(13)(14)(15). Although the risk of developing TB is reduced by $70 \%-90 \%$ among HIV-infected persons receiving HAART, compared with untreated individuals (16). However, TB risk is high following HAART initiation(17)(18).

Higher risk may be partially due to incomplete immune restoration and "unmasking" of previously undiagnosed TB in the setting of immune reconstitution(19). The effect of HAART on TB incidence in resource constrained country may be adversely affected by multiple factors including delayed presentation to care and a higher incidence of co-occurring infectious and non-infectious conditions such as undernutrition and adherence level(13)(19)(20)(20)(20).

HIV-infected children are most at risk to develope tuberculosis. Globally, from estimated 10.0 million new TB infection, 1 million of them were children less than 15 years age, in 2017. (20).In Ethiopia, reported number of PLHIV treated with HAART were $71 \%$ and out of this $32 \%$ have suppressed viral load measurement in 2017(21). TB case notification for TB was $6 \%$ with rapid diagnostic tool while TB preventive treatment reached for $47 \%$ (22). Routine screening and treatment for latent TB infection (LTBI) has been shown to reduce the risk of active TB in HIV-infected patients(23).

As to our understanding, studies on the possible effect of HAART on HIV-associated TB remains limited. We are aware that there are few studies in Ethiopia that tried to see the incidence of TB in the same population(24)(25)(6). However, their analysis was flawed. We estimated effect of HAART on the incidence of TB among HIV infected children using marginal structural regression modeling after adjusting and weighting baseline and time varying covariates.

\section{Methods}

\section{Study design and Setting}

A retrospective cohort study was conducted. We reviewed patient chart, clinical record and ART data base for five years from 2009 to 2014. The study was conducted in selected ART clinics found in southwest Ethiopia. South West Ethiopia encompasses five zones namely, Jimma, Illu Ababora, Kafa, Sheka and Bench-Maji.

\section{Definitions}

- In our study we defined Tuberculosis case as: (1) Smear positive result of acid fast bacilli or (2) TB confirmed by culture or (3) Clinical sign and symptoms compatible with TB infection criteria by WHO or (4) Chest radiography confirmation.

- Children on HAART was defined as those who took combination therapy of 3 antiretroviral drugs that included two non-nucleoside reverse transcriptase and one protease inhibitor. 
- Children, who were lost, died, or transferred out or did not develop the events until the last visit were considered as censored.

\section{Source and study population}

All children younger than 15 years having a follow up care in ART clinic in south west Ethiopia were source population. While all randomly selected HIV positive under 15 years old children registered from September, 2009 to August, 2014 in south west Ethiopia were study population.

- Exposure- treatment with highly active antiretroviral treatment (HAART) for at least 2 month

- Outcome-TB illness

\section{Inclusion and exclusion criteria}

- Inclusion- All under 15 years old children on HAART or Pre-HAART follow up who were registered from September 2009 to August 2014.

- Exclusion - All under 15 years old children who started anti-TB treatment at the beginning of follow up and/or diagnosed as TB patient

\section{Sample size and Sampling procedure}

844 sample size was calculated with double population proportion formula by considering the following assumption

$a=$ Type one error (0.05)

$\mathrm{Z} \mathrm{a/2=} \mathrm{Critical} \mathrm{value} \mathrm{at} 95 \%$ level of significance

$Z 1-\beta=$ standard normal distribution value corresponding to power $(90 \%)$

Ration $=1: 1$

Fresh list of hospitals in each zone namely Bench Maji, kefa ,Sheka, Jimma and llubabor were prepared. After having the list of ART clinics that provide ART service for less than 15 years of age, and with proportional allocation methods, samples were selected from each ART clinic with systematic random sampling technique for both ART and Pre ART cohort.

\section{Data collection tool and procedure}

A standardized tool which has been adapted from existing literature was used. Adapted tools was translated to local language by language expertise. Then relevant data was collected from patients' preART and ART follow up log books, Data base and other clinical records. Data was collected by six data clerk who work in ART clinic after receiving 2 days training on how to extract data from these records. To assure quality of data extraction, a pretest was conducted in $5 \%$ of the sampled population. 


\section{Data entry and Analysis}

The collected Data were coded and double entry was made in EpiData version 3.1 statistical package. Then the data were exported to the Statistical Package for the Social Sciences (SPSS) version 23 software for analysis. Before analysis, the data were processed and cleaned by running frequency, sorting and cross tabulation to check completeness, outliers and data entry errors. Descriptive statistics analysis were done to describe the characteristics of the study subjects.

To compare proportions of TB between HAART naïve and HAART cohort, we used the chi-square test, whereas we compared medians by the Mann-Whitney test. The Incidence rate was calculated with an Open Epi software and were expressed per 100 child-years of follow-up on HAART. The TB free survival probability were constructed by the Kaplan-Meier method and compared between HAART naïve and HAART groups by the log-rank test. The comparisons was two- tailed with $p$ values $<0.05$.

We estimated the effect of HAART on TB incidence by adjusting for confounders measured at baseline and time-varying intermediates. We did this by fitting weighted pooled logistic regression model to construct stabilized inverse probability of treatment and censoring (IPTC) weights. Then, these IPTC weights were used in a weighted pooled logistic regression model to approximate the parameters of a marginal structural Cox model(26). Each patient in the above logistic models received a time-varying weight inversely proportional to the estimated probability of having his/her own observed history of HAART initiation, as described elsewhere. For each child and visit, the denominator of the weight can be viewed as the probability that they received their actual treatment history and remained uncensored up to that time, conditional on their past treatment and covariate history. Confounders were selected based on previous studies. The covariates included in these models were age, sex, baseline clinical stage and baseline CD 4 as baseline covariates. The time varying covariates were CD4, Clinical stage, follow-up status, adherence and CPT.

Bivariable and multivariable Cox proportional hazard regression models were used to see independent predictors of TB incidence. Variables with $\mathrm{p}$-value $<0.2$ in bivariable analysis were transferred to Multivariable cox proportional hazard model. In Multivariable analysis, Variables with P-value $<0.05$ at $95 \%$ confidence level was considered as statistically significant predictors of TB incidence. The results were expressed as hazard ratios (HRs) with 95\% confidence intervals $(\mathrm{Cl})$.

\section{Results}

From the total sample (844) around $94.8 \%$ children were retrospectively followed. Half of them, $50 \%$ were HAART naïve while $50 \%$ were HAART initiated. The median follow up period for HAART naïve was 47 months with IQR of 31 months whereas HAART initiated were followed for a median of 53 months with IQR of 32 months. The total follow up period for HAART naïve children and HAART initiated were 1469.08 and 1473.91 child year time respectively. The median and inter quintile range (IQR) of age for HAART cohort were 9 and 6 years respectively. The corresponding values for the Pre-ART cohort were 7 and 5 
years respectively. In terms of gender males constitute the largest proportion in both HAART (51\%) and HAART naïve (56.8\%) groups.

During a total of 2942.99 person-years follow-up, 506 Ols occurred in 248 children (31\%). The most common reported Ols were Pneumonia (22\%) and TB (29.1\%). Nearly $2 \%$ children died, $7.8 \%$ transferred out, $12.3 \%$ lost to follow up. Of $12.3 \%$ lost to follow up $35.7 \%$ were female, and the rest $64.3 \%$ were male. Sixty five percent of the study subjects were either in WHO clinical stage I or II, while $65.3 \%$ had CD4 count above threshold at enrollment.

Baseline characteristics, children on HAART compared to those HAART naïve later had a degree of advanced WHO clinical stage $(p<0.001)$. Children who had a baseline cd4count below threshold, had a more advanced HIV clinical stage $(p=0.03)$, and were more likely to have Tuberculosis $(p<0.01)$. Although those who initiated HAART had no difference as compared to those who did not regarding gender $(p=$ $0.10)$, there was a difference regarding the medians of age across categories of HAART treatment $(p<0.001)$. The characteristics of study subjects are indicated in Table 1.

Table 1 Characterstics of HAART initiated and HAART naïve children

Time period Characteristics

Category

HAART naïve

HAART initiated

\begin{tabular}{|c|c|c|c|c|c|c|}
\hline & & & $\mathrm{TB}(\mathrm{No})$ & TB (yes) & $\mathrm{TB}(\mathrm{No})$ & TB (yes) \\
\hline \multirow[t]{12}{*}{ Baseline } & Age & $<1$ & $3(27.3 \%)$ & $8(72.7 \%)$ & $0(0 \%)$ & $18(100 \%)$ \\
\hline & & $1-3$ & $56(59.6 \%)$ & $38(40.4 \%)$ & $21(48.8 \%)$ & $22(51.2 \%)$ \\
\hline & & $3-5$ & $45(81.8 \%)$ & $10(18.2 \%)$ & $41(67.2 \%)$ & $20(32.8 \%)$ \\
\hline & & $5-15$ & $176(73.3 \%)$ & $64(26.7 \%)$ & $225(80.9 \%)$ & $53(19.1 \%)$ \\
\hline & Sex & Male & $148(65.2 \%)$ & $79(34.8 \%)$ & $127(62.3 \%$ & $77(37.7 \%)$ \\
\hline & & Female & $132(76.3 \%)$ & $41(23.7 \%)$ & $160(81.6 \%)$ & $36(18.4 \%)$ \\
\hline & WHO clinical stage & I & $158(75.2 \%)$ & $52(24.8 \%)$ & $152(78.8 \%)$ & $41(21.2 \%)$ \\
\hline & & II & $106(74.1 \%)$ & $37(25.9 \%)$ & $95(70.9 \%)$ & $39(29.1 \%)$ \\
\hline & & III & $16(34.0 \%)$ & $31(66.0 \%)$ & $34(57.6 \%)$ & $25(42.4 \%)$ \\
\hline & & Iv & $0(0 \%)$ & $0(0 \%)$ & $6(42.9 \%)$ & $8(57.1 \%)$ \\
\hline & $\mathrm{CD} 4$ & Below threshold & 73(62.9\%) & $43(37.1 \%)$ & $103(65.2 \%)$ & $55(34.8 \%)$ \\
\hline & & Above threshold & $207(72.9 \%)$ & $77(27.1 \%)$ & $184(76.0 \%)$ & $58(24.0 \%)$ \\
\hline
\end{tabular}


WHO-world health organization

TB-Tuberculosis

During the 2942.99 person-years of follow-up, 189 children developed TB.The overall TB incidence rate was 7.917 per 100 child years $(95 \% \mathrm{Cl}, 6.933-9.002)$. Whereas among HAART 7.667 per 100 -years (95\% $\mathrm{Cl}, 6.318-9.217)$ and 8.1686 per 100 person-years (95\% Cl 6.772-9.767) for HAART naïve. The medians of time to event observation for children who started HAART was statistically similar with the medians observed for untreated children ( $p=0.229)$. In comparison the proportion of HAART naïve children who developed TB (29.5\%) were higher than the $17.8 \%$ of HAART-initiated. However, no significant statistical difference was observed in terms of TB incidence between children on HAART and HAART naïve $(p=0.641)$.

The risk of TB was higher among children whose CD 4 cell count was below threshold as compared to those the corresponding value was above threshold $(p=0.003)$. However, there was similar distribution of CD4 count between children who developed TB and who does not $(p=0.744)$. The incidence of TB has a direct relationship with age, as the age increase the risk also increases $(p<0.001)$.

An unadjusted model that did not account for any confounder estimated no protective effect of HAART on TB incidence, relative to HAART naïve(HR 1.019, 95\% Cl 0.788-1.318, $\mathrm{P}=0.885)$. The unweighted model that adjusted baseline confounder suggested (HR 0.933, 95\% $\mathrm{Cl} 0.98-0.712-1.224, \mathrm{P}=0.618$ ). Similarly when we fit weighted model appropriately accounting for base line confounder the result does not suggest protective effect of HAART (HR, 0.903, 95\% Cl: 0.657-1.240, $\mathrm{P}=0.529)$. However, the model that accounted both the baseline and time- varying confounders using marginal structural models showed that HAART reduces the risk of tuberculosis; the HR from IPTC-weighted model was (HR, 0.642 , $95 \% \mathrm{Cl}, 0.442-0.931, \mathrm{P}=0.020)$ Table 2.

Table 2: Estimated effect of HAART on TB incidence among 800 HIV-infected children initiating HAART, southwest Ethiopia, 2010-2014. 


\begin{tabular}{llll}
\hline Model & HR & $95 \%$ CI & p-value \\
\hline Unweighted and unadjusted & 1.019 & $0.788-1.318$ & 0.885 \\
\hline Unweighted but adjusted & 0.933 & $0.712-1.224$ & 0.618 \\
\hline Baseline Weighted and stabilized & 0.903 & $0.657-1.240$ & 0.529 \\
\hline Time-varying Weighted and stabilized & 0.683 & $0.490-0.952$ & 0.024 \\
\hline Total Weighted and stabilized & 0.642 & $0.442-0.931$ & 0.020 \\
\hline
\end{tabular}

\section{Discussion}

This study estimated the effect of HAART on TB incidence in HIV infected children on HAART. The finding showed that HAART noticeably had a protective effect against Tuberculosis among HIV infected children in Southwest Ethiopia. Using a marginal structural model, we estimated that HAART reduced the hazard (rate) of TB incidence by $36 \%$ relative to HAART naïve. Although our methods of analysis was different, this finding was supported by a study that estimated TB incidence among children after HAART initiated(27)(28)(29). Our finding was in line with the effect observed with similar methods of analysis, between adults in Europe and the United States(30). The similarity of these findings offers evidence that HAART is effective against TB in HIV-infected children in SWE. This agreement is important because virtually all children are believed to receive HAART around the world, because of scale-up of antiretroviral provision.

The effect of HAART estimated in our study was lower than the effect revealed by similar studies in in high income countries(31)(32). This different could be resulted from the fact that, Pediatric HIV programs in Southwest Ethiopia like sub- Saharan Africa, often face challenges that could adversely increase TB among HIV infected children, including limited diagnostic capacity, delayed healthcare seeking and poor retention in care and under-nutrition(33). These factors underline why a similar protective effect of HAART on TB in children could not be expected across different settings.

The incidence of tuberculosis among children on HAART in this study was 3.59 per 100 child-years and for HAART naïve group was 4.63 per 100Child-years. Mathematically it seems low, however there is no statistically significant difference between the two groups. This finding is different from studies that supported reduced incidence of tuberculosis among HAART initiated(34) (14)(35)

In the present study high TB incidence was observed among CD4+ count strata below threshold for age during follow-up period. The result depicted an opposite relationship between duration on HAART and TB incidence. this finding is consistent with other study, and this may be due to better TB-specific immune repair with time spent on HAART(36) - Furthermore, in the first few months after HAART initiation we indicated highest rates of TB incidence, possibly due to "unmasked" infection, in the first three months after HAART initiation. Besides, the persistent high rate of new TB infections even after 24 months of HAART initiation were clearly indicated in published studies(37). We demonstrated that the high TB 
incidence rates observed in this study may be due to high ongoing community level TB transmission. Poor knowledge of the community and poor health care access. Most importantly this might be justified with our selection criteria. We selected cases with all diagnostic criteria including clinical criteria. The criteria might be less specific but highly sensitive.

Our study has some limitations. In our selection criteria we suspected that we imposed unintentional classification of prevalent tuberculosis as incident tuberculosis. Children who received no screening for tuberculosis in their enrolment were followed and if in the mean time TB was developed then it was considered as incident cases. Besides HAART was not randomly assigned, and thus the possibility of residual confounding cannot be ruled out. We also acknowledge that using Clinical TB diagnostic criteria was the limitation of our study. We were forced to use this criteria as a result of scares resource for diagnosis in the study area. This was most common practiced diagnostic criteria to initiate anti TB treatment.

\section{Conclusions}

The effect of HAART on TB incidence among HIV infected children in this study is the first valid estimate, to our knowledge, of the extent to which therapy decreases TB incidence in a highly relevant population of HIV- infected children living in Southwest setting. Tuberculosis incidence in the study area was relatively high enough to be a public health problem.

\section{Recommendation}

ART program managers and coordinators shall improve and strengthen the program. By designing strategies and tactical approach that address lost to follow up and drop, TB screening and diagnostic capacity and adherence level

\section{Declarations}

\section{Ethics approval and informed consent}

Ethical clearance was obtained from the Institutional Review Board (IRB) of Mizan-Tepi University. Permissions was obtained from Bench Maji, kefa ,Sheka, Jimma and llubabor Zonal Health Department. Moreover, a Verbal permission was obtained from selected facility administration before starting data collection. Informed Written consent was obtained from parents or legal guardian during the period of data collection.

\section{Consent for publication}

Not applicable

\section{Availability of data and materials}


The datasets during and/or analysed during the current study available from the corresponding author on reasonable request

\section{Competing interest}

We have declared that we do have neither financial nor non financial interest.

\section{Funding}

Mizan Tepi university has sponsored this study. The university has involved on supervising the data collection process. Additionally, in evaluating the proposal and the research report.

\section{Competing interest}

We have declared that we do have neither financial nor non financial interest.

\section{Authors' contribution}

Mr. Firew Tiruneh, the principal investigator, has prepared the protocol, designed the study, supervised the data collection, conduct the analysis and critically reviewed the manuscript.

Mr. Yared Deyas, co-investigator, assisted in data collection and prepared the manuscript.

\section{Acknowledgment}

First and foremost, our heartfelt gratitude goes to our colleagues for their unreserved valuable comment and contribution to this research result, with all great passion of dedication and commitment.

We are also very grateful to Department of Midwifery, college of Health Science, Mizan Tepi University for giving chance to do this protocol and willingness for funding.

We would like to extend our sincere thanks to chief executive directors and Data clerks of Mizan-Tepi teaching, Tepi district, Metu karl and Jimma medical center Hospitals for their collaboration

\section{References}

1. World health organization. Global status report on noncommunicable diseases [Internet]. 2014 [cited 2018 Aug 3]. Available from: https://www.who.int/nmh/publications/ncd-status-report-2014/en/

2. Clinical S, Diseases I, Dec N. Incidence of Tuberculosis among HIV-Infected Patients Receiving Highly Active Antiretroviral Therapy in Europe and North America Author ( $s$ ): The Antiretroviral Therapy Cohort Collaboration Linked references are available on JSTOR for this article: Incidence of Tuberculosis among HIV-In Patients Receiving Highly Active Antiret Therapy in Europe and North America. 2018;41(12):1772-82. 
3. World health organization. GLOBAL TB REPORT 2016 WITH HIV + TB [Internet]. 2016 [cited 2018 Apr 9]. Available from: https://apps.who.int/medicinedocs/en/m/abstract/Js23098en/

4. World health organization. TB fact sheet [Internet]. 2018 [cited 2020 Jan 8]. Available from: https://www.who.int/

5. Centers for Disease Control and Prevention. TB in specific populations [Internet]. [cited 2019 Mar 3]. Available from: https://www.cdc.gov/ tb/topic/populations/default.htm

6. Beshir MT, Beyene AH, Tlaye KG, Demelew TM. Incidence and predictors of tuberculosis among HIVpositive children at Adama Referral Hospital and Medical College, Oromia , Ethiopia: a retrospective follow-up study. 2019;1-8.

7. Time to act. Save a million lives by 2015. Prevent and treat tuberculosis among people living with HIV.

8. Venturini E, Turkova A, Chiappini E, Galli L, Martino M De, Thorne C. Tuberculosis and HIV coinfection in children. 2014;14(Suppl 1):1-10.

9. Reddi A, Leeper SC, Grobler AC, Geddes R, France KH, Dorse GL, et al. Preliminary outcomes of a paediatric highly active antiretroviral therapy cohort from KwaZulu-Natal , South Africa. 2005;13:113.

10. Mu W, Zhao Y, Sun X, Ma Y, Yu L, Liu X, et al. AIDS Care: Psychological and Socio-medical Aspects of AIDS / HIV Incidence and associated factors of pulmonary tuberculosis in HIV-infected children after highly active antiretroviral therapy ( HAART ) in China: a retrospective study. 2014;(February 2015):37-41.

11. Lawn SD, Wood R, De Cock KM, Kranzer K, Lewis JJ CG. Antiretrovirals and isoniazid preventive therapy in the prevention of HIV-associated tuberculosis in settings with limited health-care resources. Lancet Infect Dis. 2010;10:489-98.

12. STOP TB Partnership. The global plan to stop TB 2006-2015.

13. Edmonds A, Yotebieng M, Lusiama J, Matumona Y, Kitetele F, Cole SR, et al. The Effect of Highly Active Antiretroviral Therapy on the Survival of HIV-Infected Children in a Resource-Deprived Setting: A Cohort Study. 2011;8(6).

14. Anígilájé EA, Aderibigbe SA, Adeoti AO. Tuberculosis, before and after Antiretroviral Therapy among HIV-Infected Children in Nigeria: What Are the Risk Factors? 2016;1-19.

15. Santoro-lopes G, Maria A, Pinho F De, Harrison LH. Reduced Risk of Tuberculosis among Brazilian Patients with Advanced Human Immunodeficiency Virus Infection Treated with Highly Active Antiretroviral Therapy. 2002;34:543-6.

16. Article M. Incidence of Tuberculosis among HIV-Infected Patients Receiving Highly Active Antiretroviral Therapy in Europe and North America. 2005;41.

17. Lawn SD, Myer L, Bekker LG WR. Burden of tuberculosis in an antiretroviral treatment programme in sub-Saharan Africa: impact on treatment outcomes and implications for tuberculosis control. AIDS. 2006;20:1605-1612. 
18. Lawn SD, Badri M WR. Tuberculosis among HIV-infected patients receiving HAART: long term incidence and risk factors in a South African cohort. .AIDS. 19:2109-2116.

19. Lawn SD, Wilkinson RJ, Lipman MC WR. Immune reconstitution and "unmasking" of tuberculosis during antiretroviral therapy. Am J Respir Crit Care Med. 177:680-685.

20. Unicef. Tuberculosis is now the leading cause of death from infectious diseases for children of all ages globally [Internet]. 2017 [cited 2018 Jan 11]. Available from: https://data.unicef.org/topic/childhealth/tuberculosis/

21. World health organization. HIV country profile [Internet]. 2017. Available from: http://cfs.hivci.org/country-factsheet.html

22. Countries H. Country profiles::

23. Akolo C, Adetifa I, Shepperd S VJ. Treatment of latent tuberculosis infection in HIV infected persons. Cochrane Database Syst Rev. 2010;CD000171.

24. Alemu YM, Awoke W, Wilder-smith A. Determinants for tuberculosis in HIV-infected adults in Northwest Ethiopia : a multicentre case - control study. 2016;

25. Ayalaw SG, Alene KA, Adane AA. Incidence and Predictors of Tuberculosis among HIV Positive Children at University of Gondar Referral Hospital , Northwest Ethiopia: A Retrospective Follow-Up Study. 2015;2015.

26. Zoe Fewell,Miguel A. Hern'an, Frederick Wolfe,Kate Tilling, Hyon Choi JACS. Controlling for timedependent confounding using marginal structural models. Stata J. 2004;4:402-20.

27. Palladino C, Bello JM, Gurbindo MD, Jose MI De, Ramos T, Gonza MI, et al. Impact of Highly Active Antiretroviral Therapy ( HAART ) on AIDS and Death in a Cohort of Vertically HIV type-1 infected children:1980-2006. 2009;25(11).

28. Nesheim SR, Kapogiannis BG, Soe MM, Sullivan KM, Abrams E, Farley J, et al. Trends in Opportunistic Infections in the Pre - and Post - Highly Active Antiretroviral Therapy Eras Among HIV-Infected Children in the Perinatal AIDS. 2015;

29. Sa M. Impact of highly active antiretroviral therapy on the morbidity and mortality in Spanish human immunodeficiency virus-infected children. 2003;22(10):863-7.

30. HIV-CAUSAL Collaboration. The effect of combined antiretroviral therapy on the overall mortality of HIV-infected individuals. AIDS. 210AD;24:123-137.

31. Kirk O, Gatell JM, Mocroft A et al. Infections with Mycobacterium tuberculosis and Mycobacterium avium among HIV-infected patients after the introduction of highly active antiretroviral therapy. EuroSIDA Study Gr JD Am J Respir Crit Care Med. 2000;162:162:865-72.

32. Badri M, Wilson D WR. Effect of highly active antiretroviral therapy on incidence of tuberculosis in South Africa: a cohort study. Lancet. 2002;359:2059-64.

33. De Baets AJ, Ramet J, Msellati P LP. The unique features of pediatric HIV-1 in sub-Saharan Africa. Curr HIV Res. PLoS. 2008;6:351-362. 
34. Walters E, Cotton MF, Rabie H, Schaaf HS, Walters LO, Marais BJ. Clinical presentation and outcome of Tuberculosis in Human Immunodeficiency Virus infected children on anti-retroviral therapy. 2008;12:1-12.

35. Yang C, Chen K, Tsai J, Lin Y, Cheng S, Wang K. The impact of HAART initiation timing on HIV-TB coinfected patients, a retrospective cohort study. 2014;1-10.

36. Martinson NA, Moultrie H, van Niekerk R et al. HAART and risk of tuberculosis in HIV-infected South African children: a multi-site retrospective cohort. Int J Tuberc Lung Dis. 2009;13(7):862-867.

37. Miranda A, Morgan M, Jamal L, Laserson K, Barreira D, Silva G, et al. Impact of Antiretroviral Therapy on the Incidence of Tuberculosis: The Brazilian Experience , 1995 - 2001. 2007;(9):1995-2001. 